\title{
The synergistic antiviral effects of GSH in combination with acyclovir against BoHV-1 infection in vitro
}

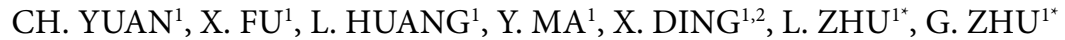

\begin{abstract}
${ }^{1}$ College of Veterinary Medicine and Jiangsu Co-innovation Center for Prevention and Control of Important Animal Infectious Diseases and Zoonoses, Yangzhou University, 48 Wenhui East Road, Yangzhou 225009, P. R. China; ${ }^{2}$ The test center of Yangzhou University, 48 Wenhui East Road, Yangzhou, 225009, P. R. China
\end{abstract}

Received December 8, 2015; accepted July 18, 2016

\begin{abstract}
Summary. - We have recently reported that bovine herpesvirus 1 (BoHV-1)-induced overproduction of reactive oxygen species (ROS) partially depends on NADPH oxidases (Noxs). In this study, we found that the decreased levels of main intracellular non-enzymatic antioxidant glutathione (GSH) during BoHV-1 infection also contributed to ROS production. Exogenous GSH administration dramatically inhibited BoHV-1 replication, indicating the critical role of decreased GSH for BoHV-1 replication. Interestingly, GSH synergistically enhanced the antiviral effects of acyclovir (ACV) against BoHV-1 infection in vitro. This study not only illuminates the effect of GSH on BoHV-1 infection but also provides evidence that pharmacological modulation of GSH-mediated ROS production in combination with specific antiviral drugs is a viable therapeutic approach to fighting virus infection.
\end{abstract}

Keywords: BoHV-1; ROS; GSH; Noxs

Bovine herpesvirus 1 (BoHV-1), an enveloped virus belonging to the alphaherpesvirus subfamily, infects cattle of all ages and breeds worldwide. Acute infection of BoHV-1 usually results in inflammatory diseases in the upper respiratory tract, nasal cavity, or ocular cavity (Jones and Chowdhury, 2007; Jones, 2009). BoHV-1-induced immune suppression initiates a polymicrobial respiratory tract disease, referred to as bovine respiratory disease complex (BRDC), which costs the US cattle industry approximately 3 billion dollars annually (Jones and Chowdhury, 2007).

Intracellular redox alterations by a variety of viruses have been extensively described in both in vitro and in vivo infection, which is associated with the progression of virus-induced

"Corresponding authors. E-mail: yzgqzhu@yzu.edu.cn (Guoqiang Zhu) or lzhu3596@163.com (Liqian Zhu); phone: (0086)-51487972590.

Abbreviations: $\mathrm{ACV}=$ acyclovir; $\mathrm{BoHV}=$ bovine herpes virus; $\mathrm{GSH}=$ glutathione; $\mathrm{MOI}=$ multiplicity of infection; $\mathrm{NAC}=\mathrm{N}-$ Acetyl-l-cysteine; Noxs = NADPH oxidases; p.i. = post infection; ROS $=$ reactive oxygen species disease by activating the synthesis of inflammatory cytokines (reviewed in Nencioni et al. (2011). Generally, the altered redox signaling leads to oxidative stress resulting from the imbalance between overproduction of ROS and the protective effect of the antioxidant system responsible for their neutralization and removal (Hu et al., 2011; Walczak-Jedrzejowska et al., 2013; Amatore et al., 2015). The main source of cellular ROS production is the family of Noxs, which consists of seven members, Nox1- to -5 and the two dual oxidases, Duox 1 and Duox2, expressed in most cell types (Bedard and Krause, 2007). The reduced GSH, a main intracellular non-enzymatic antioxidant, exerts an efficient buffering role against ROS (Bindoli et al., 2008). Our recent report has demonstrated that BoHV-1-induced ROS production contributes to both viral replication and mitochondrial damage, and the increase in ROS is partially mediated by Noxs (Zhu et al., 2016). In this study, we demonstrated that BoHV-1 infection decreased intracellular GSH levels and administration of GSH strongly impaired the replication of BoHV-1, confirming the important role of low GSH levels in the life cycle of this virus.

To assess the possible effect of cellular GSH on BoHV-1induced ROS production, confluent MDBK cells (kindly 
provided by Dr. Leonard J. Bello, University of Pennsylvania) in 24-wells plates were treated with GSH (Beyotime Biotechnology, Jiangsu, China) at optimal concentrations of 10 or 20 $\mathrm{mmol} / \mathrm{l}$, and infected with BoHV-1 (Colorado 1 strain, kindly provided by Dr. Leonard J. Bello) $(\mathrm{MOI}=1)$ at $37^{\circ} \mathrm{C}$ for $24 \mathrm{hr}$. The treatment of virus-infected cells with ROS scavenger NAC (N-Acetyl-1-cysteine) at concentrations of 1 and $5 \mathrm{mmol} / \mathrm{l}$ was used as a positive control. The level of intracellular ROS was

(a)
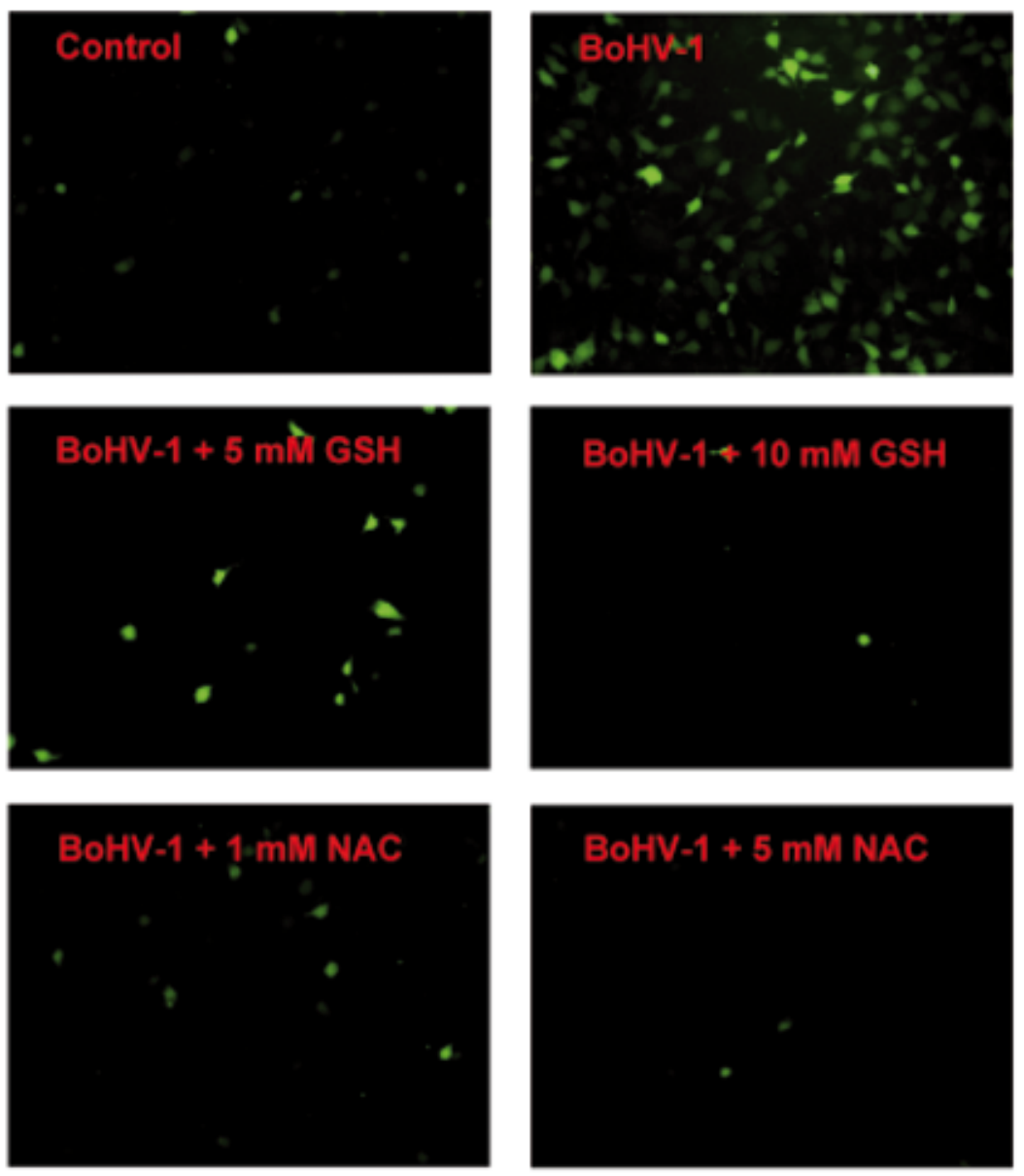

(b)

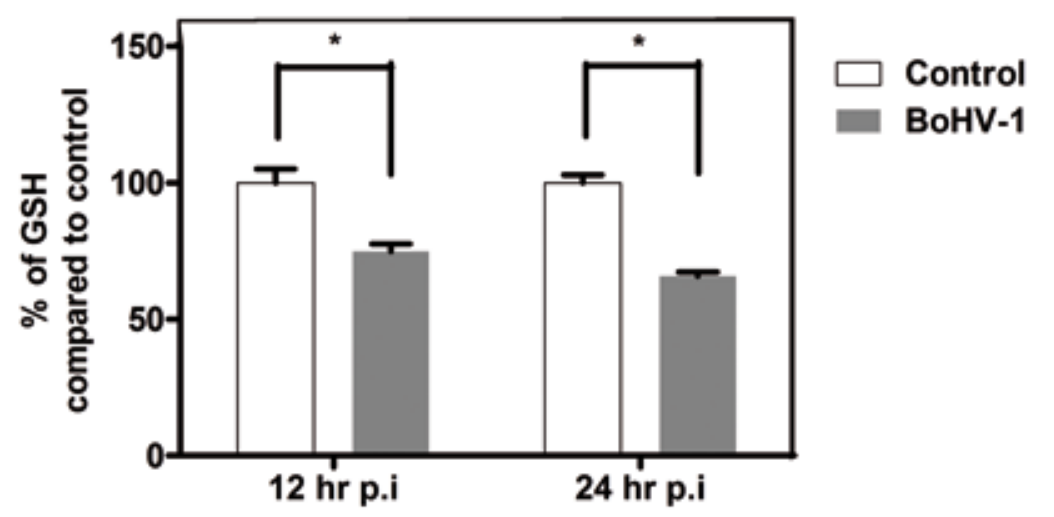

Fig. 1

The effect of GSH on BoHV-1-induced cellular ROS production

(a) ROS levels in MDBK cells treated with or without chemicals were determined using H2DCFDA ( $5 \mu \mathrm{mol} / 1$, for 30 min) following BoHV- 1 infection for $24 \mathrm{hr}$. (b) Effect of BoHV-1 on GSH in MDBK cells. MDBK cells were infected with BoHV-1 at MOI of 1 for 12 and 24 hr, then the concentration of GSH was determined. Concentrations of GSH from the virus-infected cells were normalized to mock infected control. Data shown are from three independent experiments. Statistical analyses were performed using Student's $t$ test ("indicates $\mathrm{P}<0.05$ vs. control). 
determined using ROS fluorescence indicator 2',7'-dichlorodihydrofluorescein diacetate (H2DCFDA) (Sigma-Aldrich, St. Louis, $\mathrm{MO}$ ), which can be converted to fluorescent compound dichlorofluorescein (DCF). The images were acquired under a fluorescence microscope (Olympus BX-51, Olympus, Tokyo, Japan). Treatment of cells with GSH at either concentration could dramatically reduce BoHV-1-induced ROS levels, and it showed similar capacity to that of ROS scavenger NAC (Fig. 1a). It suggested that addition of exogenous GSH inhibited cellular ROS production.

We further investigated whether BoHV-1 infection altered cellular GSH levels. Confluent MDBK cells in 24-well plates
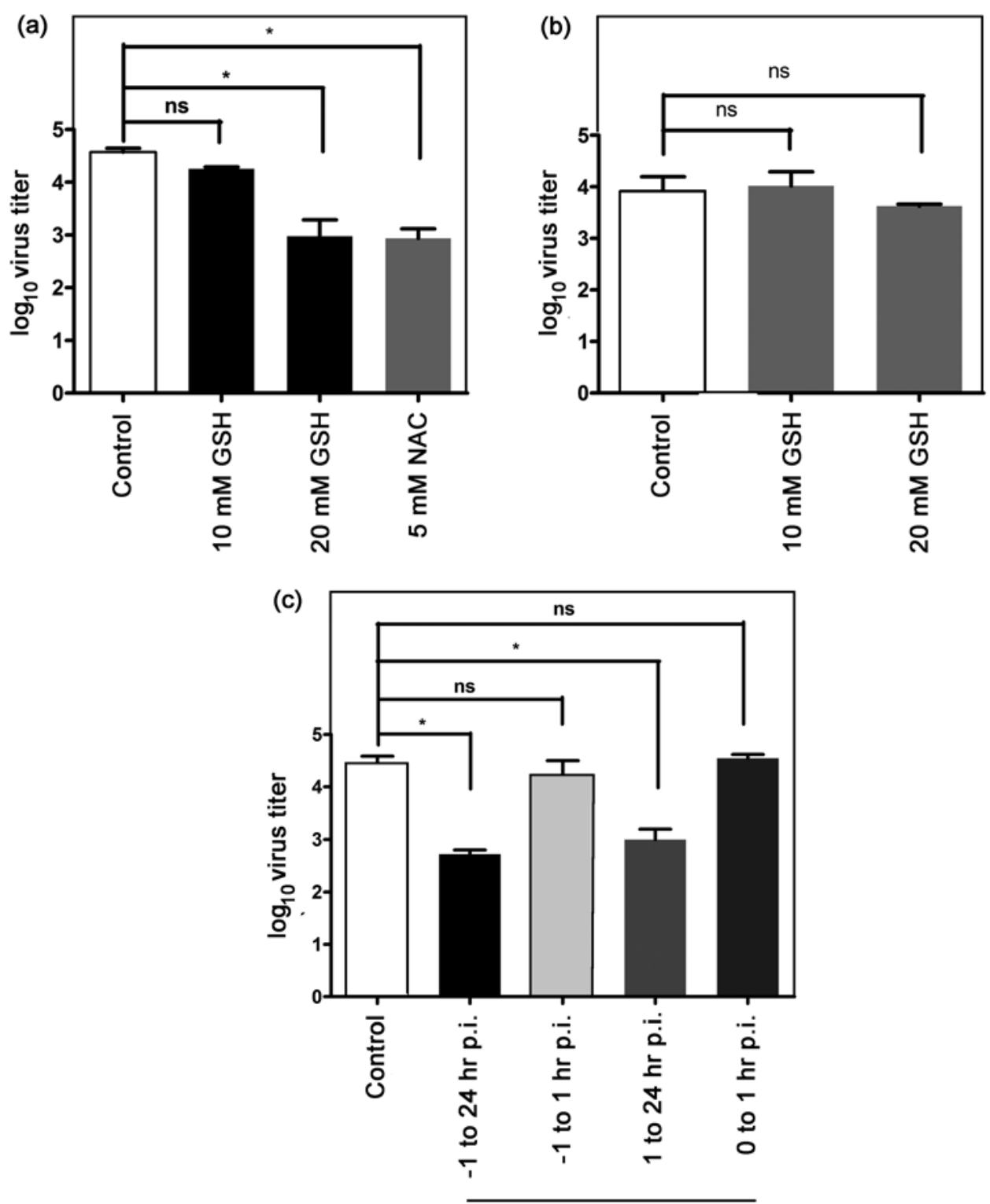

Treatment

Fig. 2

Antiviral activity of GSH against BoHV-1 infection in MDBK cells

(a) MDBK cells were mock-treated with medium or treated with chemicals at indicated concentrations, and subjected to BoHV-1 infection at an MOI of 1 for $1 \mathrm{hr}$ with or without treatment of chemicals. After extensive washing with PBS, cells were further cultured for $24 \mathrm{hr}$ with the medium containing chemicals or the vehicle. The virus yields were then titrated by TCID $_{50}$ assay. (b) Virus stocks were exposed to GSH at various concentrations or solvent at $37^{\circ} \mathrm{C}$ for $1 \mathrm{hr}$. Subsequently, the viruses were titrated by the $\mathrm{TCID}_{50}$ assay in MDBK cells. (c) MDBK cells infected with BoHV-1 at an MOI of 1 were

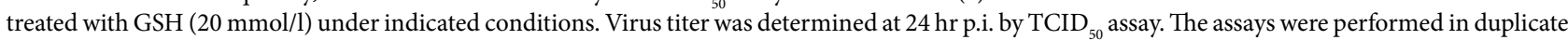
and data represent means \pm SD. ${ }^{*} \mathrm{P}<0.05$ 
were mock-infected or infected with BoHV-1 at MOI of 1, and at 12 and $24 \mathrm{hr}$ post infection (p.i.) the intracellular GSH was assayed with commercially available GSH detection kit (Beyotime Biotechnology, Jiangsu, China) completely following the manufacturer's specifications. Colorimetric determination was conducted using a multifunctional Microplate Reader (SpectraMax M2, MDC). The results were expressed as $\%$ of the control by comparison of GSH concentrations in BoHV-1-infected cells to that of the mock-infected control. As a result, BoHV-1 infection significantly decreased GSH levels to $74.88 \pm 4.02 \%$ and $65.77 \pm 2.10 \%$, respectively (Fig. 1b). It supported our assumption that BoHV-1 infection leads to GSH depletion in favor of ROS production, and which also correlated with the data that replenishment of exogenous GSH decreased ROS production (Fig. 1a).

Given the drop of GSH following BoHV-1 infection contributed to ROS production, we investigated the role of GSH depletion in BoHV-1 life cycle. Confluent MDBK cells in 24-well plates were infected with BoHV-1 $(\mathrm{MOI}=1)$ in the presence of GSH at concentrations of 10 and $20 \mathrm{mmol} / \mathrm{l}$. ROS scavenger NAC was introduced as a positive control, as it is known to inhibit BoHV-1 replication from our previous data. At $24 \mathrm{hr}$ p.i., the viral yields were determined through MDBK cells with results expressed as $\mathrm{TCID}_{50}$. The treatment of MDBK cells with GSH impaired the progeny virus yield in a dose-dependent manner (Fig. 2a). Among the detected concentrations, GSH at the higher concentration of $20 \mathrm{mmol} / \mathrm{l}$ showed neither cytotoxic effect on MDBK cells nor virucidal effect on the viral particles (data not shown and Fig. 2b). Therefore, we concluded that the depletion of cellular GSH by BoHV-1 is critical for viral replication in vitro.

To pinpoint which step of BoHV-1 replication was affected by GSH administration, confluent MDBK cells in 24-well plates were treated with GSH at a concentration of $20 \mathrm{mmol} / \mathrm{l}$ under various conditions including the early binding and entry stages (treatment: from -1 to $1 \mathrm{hr}$ p.i and from 0 to $1 \mathrm{hr}$ p.i.), post-binding entry stages (treatment: 1 to $24 \mathrm{hr}$ p.i.) as well as the whole infection stages plus a pretreatment (treatment: from -1 to $24 \mathrm{hr}$ p.i.). As showed in Fig. 2c, the virus yields were significantly reduced when the cells were treated with GSH at time duration from - 1 to $24 \mathrm{hr}$ p.i. and from 1 to $24 \mathrm{hr}$ p.i., respectively. It indicated that GSH administration mainly affected the post-entry or later stages of BoHV-1 replication, which correlated with the finding that at both 12 and $24 \mathrm{hr}$ p.i. the level of cellular GSH significantly dropped (Fig. 1b). It suggested that low levels of cellular GSH were in favor of BoHV-1 replication at the post-entry steps.

ROS has been proposed to regulate numerous cellular signaling pathways. Among them, MAPK family has been evidenced to be one of the major down-stream target molecules (Giannoni et al., 2005). Since signaling of MAPK fam- ily usually regulates both virus replication and virus-induced expression of inflammatory cytokines, such as in influenza infection (reviewed by Pleschka, 2008). Inhibition of ROS is therefore widely accepted as a potential therapeutic target for novel antiviral strategy (Nencioni et al., 2011). Here, we found that treatment of cells with GSH impaired not only ROS production but also the virus yield (Fig. 1a and Fig. 2). It may represent a potential antiviral agent. As acyclovir is commonly used to treat herpesvirus infections through suppressing viral DNA synthesis (Wuest et al., 2011), we wondered whether GSH would enhance the antiviral effect of ACV when used in combination. Therefore, confluent MDBK cells of in 24-well plates were infected with BoHV-1 at MOI of 1 in the presence of ACV at various concentrations or in combination with GSH $(10 \mathrm{mmol} / \mathrm{l})$. Apparent inhibitory effect was not observed following individual application of neither $10 \mathrm{mmol} / \mathrm{l}$ of GSH nor $10 \mu \mathrm{mol} / \mathrm{l}$ of ACV. When used in combination, ACV at both concentrations (10 and $50 \mu \mathrm{mol} / \mathrm{l}$ ) exhibited strong inhibitory effect. Especially, the synergistic effect is much more distinguished for ACV at higher concentration of $50 \mu \mathrm{mol} / \mathrm{l}$ promoted by GSH (Fig. 3). The combination of ACV and GSH would not lead to apparent cytotoxicity as determined by MTT assay (data not shown). It implied that GSH in combination with ACV could synergistically enhance the antiviral effects of $\mathrm{ACV}$ against BoHV-1 infection in vitro.

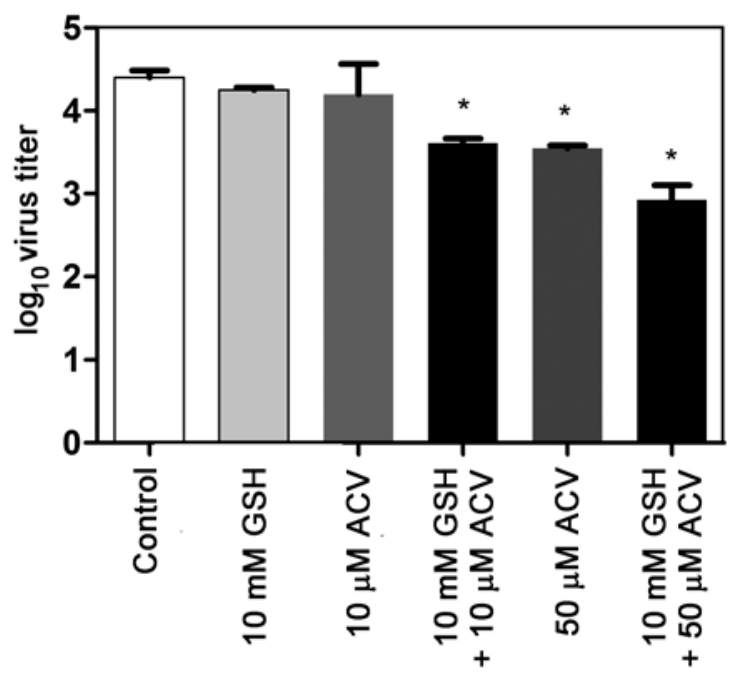

Fig. 3

GSH enhanced the antiviral effect of ACV

MDBK cells mock-treated with medium or treated with individual or combined chemicals at indicated concentrations, and subjected to BoHV-1 infection at an MOI of 1 for $1 \mathrm{hr}$ with or without treatment. After extensive washing with PBS, cells were further cultured for $24 \mathrm{hr}$ with the medium containing corresponding chemicals or the vehicle. The virus yields were then titrated by TCID $_{50}$ assay. Data shown are representative of three independent experiments. Statistical analyses were performed using Student's $t$ test, $\left({ }^{*} \mathrm{P}<0.05\right)$. 
Targeting host factors important for viral replication and disease represents a viable approach for the development of antiviral drugs, such as for the generation of novel anti-influenza drugs (Shaw, 2011). Accumulating studies suggested that intracellular redox signaling is a potential therapeutic target for novel antiviral strategy (Nencioni et al., 2011; Vlahos and Selemidis, 2014). The mechanisms underlying ROS production induced by BoHV-1 remain to be fully elucidated. Our recent report indicated that BoHV-1-induced ROS production partially depends on Noxs (Zhu et al., 2016). Here, we further reported that the virus also manipulated the main intracellular non-enzymatic antioxidant to facilitate ROS production as well as the viral replication (Fig. 1a and $2 \mathrm{a}$ and c). It is reasonable that BoHV-1 may deliberately control Noxs for ROS production, and depress cellular antioxidants to sustain a high level of intracellular ROS.

In this study, we revealed for the first time that BoHV-1 infection decreased cellular GSH, which is a potential mechanism to mediate ROS production, and evidenced that GSH administration could not only individually inhibit viral replication but also synergistically enhance the antiviral effects of $\mathrm{ACV}$ when used in combination. For clinical treatment, drug combinations are generally considered to get much more extensive protective effect. Thus, pharmacological modulation of GSH-mediated ROS production in combination with specific antiviral drugs is a viable therapeutic approach to fighting virus infection.

Acknowledgements. The authors thank Dr. Leonard J. Bello, University of Pennsylvania, for providing the MDBK cells and the Colorado 1 strain of BoHV-1. This research was supported by Chinese National Science Foundation (Grant No. 31472172), and partially by the Priority Academic Program Development of Jiangsu Higher Education Institutions (PAPD), and Science and Technology Department of Jiangsu province (Grant No. BE2014358).

\section{References}

Amatore D, Sgarbanti R, Aquilano K, Baldelli S, Limongi D, Civitelli L, Nencioni L, Garaci E, Ciriolo MR, Palamara AT (2015): Influenza virus replication in lung epithelial cells depends on redox-sensitive pathways activated by NOX4derived ROS. Cell. Microbiol. 17, 131-145. http://dx.doi. org $/ 10.1111 / \mathrm{cmi} .12343$

Bedard K, Krause KH (2007): The NOX family of ROS-generating NADPH oxidases: physiology and pathophysiology.
Physiol. Rev. 87, 245-313. http://dx.doi.org/10.1152/ physrev.00044.2005

Bindoli A, Fukuto JM, Forman HJ (2008): Thiol chemistry in peroxidase catalysis and redox signaling. Antioxid. Redox Signal. 10, 1549-1564. http://dx.doi.org/10.1089/ars.2008.2063

Giannoni E, Buricchi F, Raugei G, Ramponi G, Chiarugi P (2005): Intracellular reactive oxygen species activate Src tyrosine kinase during cell adhesion and anchorage-dependent cell growth. Mol. Cell. Biol. 25, 6391-6403. http://dx.doi. org/10.1128/MCB.25.15.6391-6403.2005

Hu S, Sheng WS, Schachtele SJ, Lokensgard JR (2011): Reactive oxygen species drive herpes simplex virus (HSV)-1induced proinflammatory cytokine production by murine microglia. J. Neuroinflammation 8, 123. http://dx.doi. org/10.1186/1742-2094-8-123

Jones C (2009): Regulation of Innate Immune Responses by Bovine Herpesvirus 1 and Infected Cell Protein 0 (bICP0). Viruses 1, 255-275. http://dx.doi.org/10.3390/v1020255

Jones C, Chowdhury S (2007): A review of the biology of bovine herpesvirus type 1 (BHV-1), its role as a cofactor in the bovine respiratory disease complex and development of improved vaccines. Anim. Health Res. Rev. 8, 187-205. http://dx.doi.org/10.1017/S146625230700134X

Nencioni L, Sgarbanti R, Amatore D, Checconi P, Celestino I, Limongi D, Anticoli S, Palamara AT, Garaci E (2011): Intracellular redox signaling as therapeutic target for novel antiviral strategy. Curr. Pharm. Des. 17, 3898-3904. http://dx.doi.org/10.2174/138161211798357728

Pleschka S (2008): RNA viruses and the mitogenic Raf/MEK/ERK signal transduction cascade. Biol. Chem. 389, 1273-1282. http://dx.doi.org/10.1515/BC.2008.145

Shaw ML (2011): The host interactome of influenza virus presents new potential targets for antiviral drugs. Rev. Med. Virol. 21, 358-369. http://dx.doi.org/10.1002/rmv.703

Vlahos R, Selemidis S (2014): NADPH oxidases as novel pharmacologic targets against influenza A virus infection. Mol. Pharmacol. 86, 747-159. http://dx.doi.org/10.1124/mol.114.095216

Walczak-Jedrzejowska R, Wolski JK, Slowikowska-Hilczer J (2013): The role of oxidative stress and antioxidants in male fertility. Cent. European J. Urol. 66, 60-67. http://dx.doi. org/10.5173/ceju.2013.01.art19

Wuest T, Zheng M, Efstathiou S, Halford WP, Carr DJ (2011): The herpes simplex virus-1 transactivator infected cell protein- 4 drives VEGF-A dependent neovascularization. PLoS Pathog. 7, e1002278. http://dx.doi.org/10.1371/ journal.ppat.1002278

Zhu L, Yuan C, Zhang D, Ma Y, Ding X, Zhu G (2016): BHV-1 induced oxidative stress contributes to mitochondrial dysfunction in MDBK cells. Vet. Res. 22, 47.http://dx.doi. org/10.1186/s13567-016-0332-2 